\title{
Impact of type 2 diabetes mellitus on coronary function evaluated by quantitative flow ratio in patients underwent percutaneous coronary intervention
}

\section{zhen ye}

Fujian Medical University Union Hospital

qin chen

Fujian Medical University Union Hospital

jiaxin zhong

Fujian Medical University Union Hospital long chen

Fujian Medical University Union Hospital lihua chen

Fujian Medical University Union Hospital mingfang ye

Fujian Medical University Union Hospital yuanming yan

Fujian Medical University Union Hospital lianglong chen

Fujian Medical University Union Hospital yukun luo ( $D$ luoyukun@hotmail.com )

Fujian Medical University Union Hospital

\section{Original investigation}

Keywords: Type 2 diabetes mellitus, Quantitative flow ratio, Coronary function, Percutaneous coronary intervention

Posted Date: May 10th, 2021

DOl: https://doi.org/10.21203/rs.3.rs-484734/v1

License: (9) This work is licensed under a Creative Commons Attribution 4.0 International License. Read Full License 


\section{Abstract \\ Background}

The co-existence of type 2 diabetes mellitus (T2DM) seems to reduce the therapeutic benefits from interventional technology in patients diagnosed with coronary artery disease (CAD). But the influence of T2DM on coronary disease still lacks the evidence from a perspective of functional assessment. Therefore, this study aimed to explore the impact of T2DM on coronary function in patients underwent percutaneous coronary intervention $(\mathrm{PCl})$ through the new functional evaluation approach that is quantitative flow ratio (QFR).

\section{Methods}

Patients who underwent successful PCl and one-year angiographic follow-up were retrospectively screened and analyzed by QFR. Based on co-presence or absence of T2DM at the time of first admission or first discharge, 677 enrolled patients (794 lesions) were classifed into DM group (211 patients, 261 lesions) and Non-DM group (466 patients, 533 lesions). The post-PCI QFR, follow-up QFR, change value of QFR and incidence of functional restenosis were compared between the two groups.

\section{Results}

The two groups reached similar levels of post-PCI QFR $(0.95 \pm 0.09$ vs. $0.96 \pm 0.06, p=0.292)$, but after one year follow-up, the degree of QFR decline in the DM group was more severe $(-0.02 \pm 0.09$ vs. $-0.01 \pm$ $0.07, p=0.023)$, leading to the follow-up QFR was lower than the Non-DM group ( $0.93 \pm 0.11$ vs. $0.96 \pm$ $0.07, p<0.001)$. What's more, the incidence of functional restenosis in the DM group increased significantly within one year $(10.0 \%$ vs. $4.7 \%, p=0.004)$.

\section{Conclusion}

T2DM can accelerate the functional decline of target coronary vessels after successful PCl.

\section{Background}

It is well-established that the co-presence of type 2 diabetes mellitus (T2DM) will increase adverse cardiovascular events in patients diagnosed with coronary artery disease (CAD). The endothelial dysfunction and microvascular disorder induced by diabetes make the patients with coronary disease face much more complicated clinical situations in treatment ${ }^{[1-3]}$. Previous studies have showed that coexistence of T2DM seems to reduce the therapeutic benefits from interventional technology. Therefore, 
there is a more stringent demand of disease assessment and management in patients who underwent percutaneous coronary intervention $(\mathrm{PCl})$ and diagnosed with T2DM ${ }^{[4,5]}$.

As a promising part of disease management, functional evaluation is of increasingly importance in patients diagnosed with CAD. Fractional flow reserve (FFR) is currently recognized as the gold standard in functional evaluation of coronary artery. Assessing severity of coronary stenosis, guiding revascularization decision, and tracing the coronary function in follow-up, make FFR become a multifunctional evaluating approach. However, the clinical application of FFR is limited for the use of dilatation drugs, pressure guide wire, long measurement time and high cost ${ }^{[6-8]}$. Quantitative flow ratio (QFR) is an emerging and less-invasive technology to compute FFR based on three-dimensional coronary artery reconstruction and fluid dynamics computation ${ }^{[9]}$. Previous landmark studies, like FAVOR pilot study, FAVOR II China study, or FAVOR II Europe-Japan study, have confirmed the accuracy of QFR compared with FFR ${ }^{[9-11]}$. In the light of no requirement of pressure wire and quicker procedural time, QFR is considered to be an alternative choice in coronary functional evaluation ${ }^{[10-13]}$.

Howbeit technologies emerge and develop rapidly, these functional evaluating methods still typically underutilized, especially in CAD patients with complications like T2DM. Meanwhile, the influence of T2DM on coronary disease still lacks the evidence from a perspective of functional assessment. In addition, the application of the new functional evaluation approach, QFR, in CAD patients with T2DM also needs further exploration. Therefore, this study aimed to explore the impact of T2DM on coronary function through QFR in patients underwent PCl.

\section{Methods}

\subsection{Study design}

Consecutive patients underwent PCI were recruited from August 2015 to March 2017 in Fujian Medical University Union Hospital. Patients underwent $\mathrm{PCl}$ and one year angiographic follow-up were eligible for enrollment when inclusion criteria were met. Indication for QFR computation was: (1) Diameter stenosis (DS) of at least one lesion between 50-90\% (visual assessment); (2) Reference vessel diameter size $\geq$ $2.5 \mathrm{~mm}$ (visual assessment). Patients consisting any of the following clinical characters would be excluded: (1) acute ST segment elevation myocardial infarction (STEMI) within 72 hours; (2) lack of follow-up data; (3) the circumstances where QFR computation couldn't be performed, including: a. only one lesion with $\mathrm{DS}<50 \%$ or $>90 \%$ and thrombolysis in myocardial infarction (TIMI) grade $<3$; b. reference vessel diameter size $<2 \mathrm{~mm}$; c. lacked of two optimal angiographic projections at least $25^{\circ}$ apart; $\mathrm{d}$. the lesion involved myocardial bridge or bypass graft; e. severe overlap or tortuosity of target blood vessels; $f$. poor angiographic image quality.

All enrolled patients were computed retrospectively for QFR, and their clinical characteristics of pre-PCl, post-PCl and one-year angiographic follow-up were collected. According to co-existence or absence of 
T2DM, patients were classified into DM group and Non-DM group. This study was approved by the Ethics Committee of Union Hospital, Fujian Medical University (No.2020KY098).

\subsection{PCl performance and QFR computation}

$\mathrm{PCl}$ was performed according to the revascularization guidelines at that time. The type and expansion of stent were determined by the experienced cardiologists relied on their own judgment. All patients received dual antiplatelet therapy for at least 12 months after $\mathrm{PCl}$.

QFR computations were performed by two trained operators who blinded to clinical data through the AngioPlus system (Pulse Medical Imaging Technology Shanghai, China) according to standard operating procedures. The 3D reconstruction of target vessel was performed based on automated contouring of two angiographic projections recorded at $15 \mathrm{frames} / \mathrm{s}$ and at least $25^{\circ}$ apart. Proximal and distal reference points were used to indicate the region of interest. After 3D reconstruction, the QFR value of target coronary artery was computed through contrast flow velocity models.

\subsection{Data collection and follow-up}

Electronic medical record system was used to retrospectively collect relevant clinical data of enrolled patients at the time of first admission and one-year angiographic follow-up. Serum biochemical results such as glucose, low-density lipoprotein cholesterol (LDL-C), creatinine, troponin I, N-terminal pro brain natriuretic peptide (NT-proBNP) and C-reaction protein (CRP) were measured in the hospital clinical laboratory using routine automated techniques. All patients were treated in accordance with the clinical guidelines recommended at the time of discharge.

Functional restenosis was defined as QFR $<0.8$ at one-year follow-up after successful PCI. Major adverse cardiac events (MACEs) were regarded as a complex of myocardial infarction, admission for heart failure, cardiogenic death and target vessel revascularization (TVR). Unless there was a clear non-cardiac cause, otherwise that all deaths were considered cardiogenic deaths. TVR was characterized by ischemia-driven percutaneous or surgical revascularization of the target blood vessel.

We recorded the incidence of functional restenosis based on results of retrospective QFR computation, and noted the number of MACEs that occurred within one year by telephone follow-up or medical records reviews.

\subsection{Statistical analysis}

Continuous variables were presented as mean \pm standard deviation for normally distributed data or as medians and interquartile range for non-normally distributed data. Categorical variables were presented as counts and percentages. Normality was tested with the Kolmogorov-Smirnov test or Shapiro-Wilk test appropriately. Comparisons between continuous variables were evaluated with Student's t test or Mann-Whitney $U$ test depending on the results of normality test. Comparisons between categorical variables were performed with Pearson's $\chi 2$ test or Fisher's exact test as appropriate. A two-sided $p$ value 
$<0.05$ was considered statistically significant. All analyses were performed with SPSS 26.0 (IBM Inc., New York, NY, USA).

\section{Results}

A total of 1016 patients (1198 lesions) were recruited in this study. After exclusion on the basis of predefined criteria, 677 patients (794 lesions) were included in the final analysis. The enrolled patients were divided into DM group (211 patients, 261 lesions) and Non-DM group (466 patients, 533 lesions) based on co-presence or absence of T2DM at the time of first admission or first discharge (Fig. 1).

\subsection{Baseline characteristics}

Co-existence of T2DM accounted for $31.2 \%$ of all enrolled patients. The Non-DM group had higher proportion of smoking $(60.3 \%$ vs. $51.2 \%, p=0.026)$ and STEMI $(21.5 \%$ vs. $14.2 \%, p=0.027)$. The DM group had higher incidence of hypertension $(72.0 \%$ vs. $59.7 \%, p=0.002)$, renal insufficiency $(6.2 \%$ vs. $2.4 \%, p=0.013)$ and previous $\mathrm{PCl}$ history $(22.3 \%$ vs. $11.4 \%, p<0.001)$. In addition, the glucose $(8.70 \pm 3.47$ vs. $5.60 \pm 1.14, p<0.001)$ and $E / E^{\prime}(14.49 \pm 5.99$ vs. $12.74 \pm 13.70, p<0.001)$ were found higher in the diabetics, while lower level of LDL-C $(2.82 \pm 1.02$ vs. $3.01 \pm 1.01, p=0.015)$ was found in these patients. No significant difference were found in age, gender, previous MI, creatinine, troponin I, NT-proBNP, CRP and left ventricular ejection fraction (LVEF) between the two groups (Table 1).

\subsection{Variation of biochemical results}

Compared to the level at first admission, glucose levels of two groups were both decreased at one-year follow-up. Further, the DM group still had a higher glucose level at follow-up $(7.39 \pm 2.66$ vs. $5.32 \pm 0.92, p$ $<0.001)$. The LDL-C levels of two groups were similar ( $2.30 \pm 1.07$ vs. $2.24 \pm 0.81, p=0.766)$ at one-year follow-up. Additionally, the diabetics had higher NT-proBNP level (100.00 (46.25-250.75) vs. 75.00 (40.00$173.25), p=0.038)$ and $E / E^{\prime}$ value (14.76 \pm 6.73 vs. $\left.12.61 \pm 5.09, p<0.001\right)$ (Table 2). 
Table 1

Baseline characteristics

\begin{tabular}{|c|c|c|c|}
\hline & $\begin{array}{l}\text { DM group } \\
(n=211)\end{array}$ & $\begin{array}{l}\text { Non-DM group } \\
(n=466)\end{array}$ & $P$ value \\
\hline Age, years & $63.57 \pm 9.50$ & $62.38 \pm 10.38$ & 0.250 \\
\hline Male, n (\%) & $168(79.6 \%)$ & $387(83.0 \%)$ & 0.283 \\
\hline Smoking history, n (\%) & $108(51.2 \%)$ & $281(60.3 \%)$ & 0.026 \\
\hline Hypertension, n (\%) & $152(72.0 \%)$ & $278(59.7 \%)$ & 0.002 \\
\hline Renal insufficiency, n (\%) & $13(6.2 \%)$ & $11(2.4 \%)$ & 0.013 \\
\hline Previous MI, n (\%) & $26(12.3 \%)$ & $42(9.0 \%)$ & 0.185 \\
\hline Previous PCI, n (\%) & $47(22.3 \%)$ & $53(11.4 \%)$ & $\varangle 0.001$ \\
\hline \multicolumn{4}{|l|}{ Disease types } \\
\hline Stable angina, $\mathrm{n}(\%)$ & $27(12.8 \%)$ & $44(9.4 \%)$ & 0.187 \\
\hline Unstable angina, n (\%) & $117(55.5 \%)$ & $244(52.4 \%)$ & 0.455 \\
\hline NSTEMI, n (\%) & $37(17.5 \%)$ & $78(16.7 \%)$ & 0.798 \\
\hline STEMI ( $\geq 72 \mathrm{~h}), \mathrm{n}(\%)$ & $30(14.2 \%)$ & $100(21.5 \%)$ & 0.027 \\
\hline \multicolumn{4}{|l|}{ Laboratory indexes } \\
\hline Glucose, $\mathrm{mmol} / \mathrm{L}$ & $8.70 \pm 3.47$ & $5.60 \pm 1.14$ & $\otimes 0.001$ \\
\hline LDL-C, mmol/L & $2.82 \pm 1.02$ & $3.01 \pm 1.01$ & 0.015 \\
\hline Creatinine, umol/L & $85.60 \pm 53.29$ & $78.97 \pm 19.60$ & 0.415 \\
\hline Troponin I, ug/L & $4.61 \pm 12.25$ & $6.75 \pm 13.79$ & 0.321 \\
\hline NT-proBNP, pg/mL & $146.00(57.50-631.50)$ & $158.00(58.00-600.00)$ & 0.893 \\
\hline CRP, mg/L & $2.27(0.78-7.48)$ & $2.72(0.86-7.85)$ & 0.411 \\
\hline LVEF, \% & $60.11 \pm 12.03$ & $60.61 \pm 10.88$ & 0.853 \\
\hline$E / E^{\prime}$ & $14.49 \pm 5.99$ & $12.74 \pm 13.70$ & $\varangle 0.001$ \\
\hline
\end{tabular}

DM, diabetes mellitus; MI, myocardial infarction; PCI, percutaneous coronary intervention; NSTEMI, nonST-segment elevation myocardial infarction; STEMI, ST-segment elevation myocardial infarction; LDL-C, low-density lipoprotein cholesterol; NT-proBNP, N-terminal pro brain natriuretic peptide; CRP, C-reaction protein; LVEF, left ventricular ejection fraction 
Table 2

Variation of biomedical indicators

\begin{tabular}{|c|c|c|c|}
\hline & $\begin{array}{l}\text { DM group } \\
(n=211)\end{array}$ & $\begin{array}{l}\text { Non-DM group } \\
(n=466)\end{array}$ & $P$ value \\
\hline \multicolumn{4}{|l|}{ Pre-PCl } \\
\hline Glucose, $\mathrm{mmol} / \mathrm{L}$ & $8.70 \pm 3.47$ & $5.60 \pm 1.14$ & $\varangle 0.001$ \\
\hline LDL-C, mmol/L & $2.82 \pm 1.02$ & $3.01 \pm 1.01$ & 0.015 \\
\hline Creatinine, umol/L & $85.60 \pm 53.29$ & $78.97 \pm 19.60$ & 0.415 \\
\hline Troponin I, ug/L & $4.61 \pm 12.25$ & $6.75 \pm 13.79$ & 0.321 \\
\hline NT-proBNP, pg/mL & $146.00(57.50-631.50)$ & $158.00(58.00-600.00)$ & 0.893 \\
\hline CRP, mg/L & $2.27(0.78-7.48)$ & $2.72(0.86-7.85)$ & 0.411 \\
\hline LVEF, \% & $60.11 \pm 12.03$ & $60.61 \pm 10.88$ & 0.853 \\
\hline$E / E^{\prime}$ & $14.49 \pm 5.99$ & $12.74 \pm 13.70$ & $\varangle 0.001$ \\
\hline \multicolumn{4}{|l|}{ Follow-up } \\
\hline Glucose, $\mathrm{mmol} / \mathrm{L}$ & $7.39 \pm 2.66$ & $5.32 \pm 0.92$ & $₫ 0.001$ \\
\hline LDL-C, mmol/L & $2.30 \pm 1.07$ & $2.24 \pm 0.81$ & 0.766 \\
\hline Creatinine, umol/L & $85.92 \pm 53.23$ & $81.45 \pm 22.90$ & 0.777 \\
\hline Troponin I, ug/L & $0.01 \pm 0.03$ & $0.01 \pm 0.01$ & 0.503 \\
\hline NT-proBNP, pg/mL & $100.00(46.25-250.75)$ & $75.00(40.00-173.25)$ & 0.038 \\
\hline CRP, mg/L & $1.13(0.50-3.81)$ & $0.88(0.43-2.47)$ & 0.051 \\
\hline LVEF, \% & $62.11 \pm 10.46$ & $62.09 \pm 10.58$ & 0.969 \\
\hline$E / E^{\prime}$ & $14.76 \pm 6.73$ & $12.61 \pm 5.09$ & $\varangle 0.001$ \\
\hline
\end{tabular}

DM, diabetes mellitus; $\mathrm{PCl}$, percutaneous coronary intervention; LDL-C, low-density lipoprotein cholesterol; NT-proBNP, N-terminal pro brain natriuretic peptide; CRP, C-reaction protein; LVEF, left ventricular ejection fraction

\subsection{QFR analysis}

After successful revascularization, the post-PCI QFR of the two groups reached a similar level $(0.95 \pm 0.09$ vs. $0.96 \pm 0.06, p=0.292$ ). Nevertheless, the DM group still experienced a more severe decline in QFR value after one year ( $-0.02 \pm 0.09$ vs. $-0.01 \pm 0.07, p=0.023$ ) (Fig. 2), which led to a lower follow-up QFR value $(0.93 \pm 0.11$ vs. $0.96 \pm 0.07, p<0.001)$. Furthermore, the incidence of functional restenosis in the $\mathrm{DM}$ 
group was found to increase significantly within one year $(10.0 \%$ vs. $4.7 \%, p=0.004)$. In addition, the follow-up blood flow resistance (BFR), which was considered as a functional indicator, was higher in the diabetics ( $32.42 \pm 50.05$ vs. $21.71 \pm 33.43, p=0.009$ ). Meanwhile, the increase of BFR was found to be higher in the DM group simultaneously $(13.40 \pm 42.34$ vs. $5.24 \pm 34.68, p=0.004)$ (Table 3 ).

Table 3

Functional evaluation

\begin{tabular}{|c|c|c|c|}
\hline & $\begin{array}{l}\text { DM group } \\
(n=261)\end{array}$ & $\begin{array}{l}\text { Non-DM group } \\
(n=533)\end{array}$ & $P$ value \\
\hline \multicolumn{4}{|l|}{ Target vessel } \\
\hline LM, n (\%) & $2(0.8 \%)$ & $9(1.7 \%)$ & 0.296 \\
\hline LAD, n (\%) & $130(49.8 \%)$ & $290(54.4 \%)$ & 0.222 \\
\hline LCX, n (\%) & $43(16.5 \%)$ & $83(15.6 \%)$ & 0.744 \\
\hline RCA, n (\%) & $84(32.2 \%)$ & $136(25.5 \%)$ & 0.049 \\
\hline Other branches, n (\%) & $2(0.8 \%)$ & $15(2.8 \%)$ & 0.061 \\
\hline Post-PCI BFR, mmHg*s/m & $19.11 \pm 34.80$ & $16.33 \pm 29.20$ & 0.871 \\
\hline Follow-up BFR, mmHg*s/m & $32.42 \pm 50.05$ & $21.71 \pm 33.43$ & 0.009 \\
\hline$\triangle \mathrm{BFR}, \mathrm{mmHg}{ }^{\star} \mathrm{s} / \mathrm{m}$ & $13.40 \pm 42.34$ & $5.24 \pm 34.68$ & 0.004 \\
\hline Post-PCI QFR & $0.95 \pm 0.09$ & $0.96 \pm 0.06$ & 0.292 \\
\hline Follow-up QFR & $0.93 \pm 0.11$ & $0.96 \pm 0.07$ & $\varangle 0.001$ \\
\hline$\triangle \mathrm{QFR}$ & $-0.02 \pm 0.09$ & $-0.01 \pm 0.07$ & 0.023 \\
\hline Functional restenosis, n (\%) & $26(10.0 \%)$ & $25(4.7 \%)$ & 0.004 \\
\hline \multicolumn{4}{|c|}{ Values are presented as the mean \pm standard deviation or $\mathrm{n}(\%)$} \\
\hline \multicolumn{4}{|c|}{$\begin{array}{l}\text { DM, diabetes mellitus; LM, left main artery; LAD, left anterior descending artery; LCX, left circumflex } \\
\text { artery; RCA, right coronary artery; PCI, percutaneous coronary intervention; BFR, blood flow resistance; } \\
\triangle B F R=\text { Follow-up BFR - Post-PCI BFR; QFR, quantitative flow ratio; } \triangle Q F R \text { = Follow-up QFR - Post-PCl } \\
Q \text { QFR }\end{array}$} \\
\hline
\end{tabular}

\subsection{Clinical outcomes}

The DM group had a higher incidence of MACEs $(14.2 \%$ vs. $6.9 \%, p=0.002)$, which was mainly attributed to the higher incidence of TVR $(11.8 \%$ vs. $5.2 \%, p=0.002)$. In addition, the incidence of stent thrombosis in the DM group was also significantly higher and comparable ( $3.8 \%$ vs. $0.6 \%, p=0.003)$ (Table 4$)$. 
Table 4

Clinical outcomes

\begin{tabular}{|llll|}
\hline & $\begin{array}{l}\text { DM group } \\
(\mathbf{n = 2 1 1 )}\end{array}$ & $\begin{array}{l}\text { Non-DM group } \\
(\mathbf{n = 4 6 6 )}\end{array}$ & P value \\
\hline MACEs, n (\%) & $30(14.2 \%)$ & $32(6.9 \%)$ & 0.002 \\
\hline MI, n (\%) & $2(0.9 \%)$ & $1(0.2 \%)$ & 0.183 \\
\hline Heart failure, n (\%) & $4(1.9 \%)$ & $8(1.7 \%)$ & 0.870 \\
\hline Cardiac death, n (\%) & $0(0 \%)$ & $0(0 \%)$ & $/$ \\
\hline TVR, n (\%) & $25(11.8 \%)$ & $24(5.2 \%)$ & 0.002 \\
\hline Stent thrombosis, n (\%) & $8(3.8 \%)$ & $3(0.6 \%)$ & 0.003 \\
\hline Values are presented as n (\%) & & \\
\hline
\end{tabular}

DM, diabetes mellitus; MACEs, major adverse cardiac events; MI, myocardial infarction; TVR, target vessel revascularization

\section{Discussion}

The main highlights of this study were as follows: (1) using the functional indicator (QFR) to evaluate the effect of T2DM on coronary function for the first time; (2) owning the compelling evidence of one-year follow-up through functional evaluation; (3) corroborating that T2DM can accelerate the functional decline of target coronary vessels after successful PCl.

In light of coronary microvascular dysfunction (CMD) is not uncommon for diabetics, functional evaluation in these patients seems to be more complex. Previous studies have showed that CMD may affect coronary hemodynamics and further change functional evaluable results. Thus, the accuracy of functional evaluation methods (i.e., QFR) in diabetics has been questioned ${ }^{[14-16]}$. However, several subgroup studies showed QFR maintains favorable performance in patients with T2DM, then dispelled the suspicion that T2DM may influence the QFR measurement ${ }^{[17-19]}$. Therefore, using QFR to evaluate coronary function in diabetics seems to be feasible and reliable.

Comparing to the non-diabetics, we found that patients co-existing T2DM had a similar level of NTproBNP and a higher level of E/E' at admission, while a higher level of NT-proBNP and E/E' at the time of one-year follow-up. It seems that co-presence of T2DM deteriorate the whole cardiac function in CAD patients. Meanwhile, the diabetics had a higher incidence of MACEs and more frequent TVR, indicating a worse cardiovascular outcome in CAD patients who co-existing T2DM. These findings are in accordance with previous researches, T2DM may accelerate the decline of cardiac function and increase cardiovascular risk ${ }^{[20,21]}$. 
Furthermore, we found the new evidence to confirm the coronary injury induced by T2DM from the functional evaluation view. The DM group reached a similar level of post-PCI QFR, albeit they had a severe coronary stenosis at admission. However, the benefits from revascularization in T2DM patients seemed to be decreased as time went on. The decline of QFR in the DM group was more obvious than the Non-DM group, leading to a lower follow-up QFR and higher incidence of functional restenosis in diabetics. The functional evaluation results showed a worse coronary recovery in CAD patients with T2DM, which is in line with the results of biomedical indicators and clinical outcomes.

The possible reasons why T2DM deteriorates the post $-\mathrm{PCl}$ coronary function are as follows: (1) Unsatisfactory glucose management: We found that the level of glucose of the DM group was higher than ideal control level at admission and one-year follow-up simultaneously. In addition, the glucose management of the DM group was presented to be worse than the Non-DM group. High glucose level is associated with activation of oxidative stress, sugar oxidation and systemic inflammatory response, which induce the damage to endothelial cells in coronary artery. As a result of inflammatory reaction, lipid deposition is promoted in coronary artery, then lipid-rich plaques are formed. Moreover, advanced glycation end products (AGE) participate in the degradation of coronary function. The coronary function is impaired by these chain reactions caused by high glucose ${ }^{[22,23]}$. (2) Insulin resistance: Insulin resistance is a typical characteristic of T2DM. It can increase circulating lipids and plasminogen activator inhibitor-1 level, then decreases the ability to decompose accumulated fibrin in coronary artery wall. Besides, insulin resistance may aggravate hypertension, which contributes to the acceleration of atherosclerosis ${ }^{[22]}$. Meantime, high insulin levels triggered by insulin resistance can induce the intimal hyperproliferation by direct growth factor-like effect ${ }^{[24]}$. (3) Higher incidence of stent thrombosis: We found that the DM group was connected with higher incidence of stent thrombosis. Meanwhile, the DM group had a higher follow-up BFR and a more obvious increase in BFR variation, which might reflect the prethrombotic state of diabetics to a certain extent ${ }^{[25]}$. (4) Microvascular dysfunction: Previous studies have showed that T2DM is related to CMD ${ }^{[26,27]}$. Coronary function can be affected by CMD to some degree, which reflects the decrease in QFR value.

The results of functional evaluation show the explicit evidence of impaired coronary function through QFR, and further confirm the deterioration in coronary function caused by T2DM. In view of functional evaluation, a more stringent demand of disease management should be advocated in CAD patients coexisting T2DM. Intensive glucose control, dyslipidemia management, strengthening balloon expansion may contribute to improvement in coronary function ${ }^{[28,29]}$. In addition, Lee et.al. found that the adverse events of diabetics after $\mathrm{PCl}$ mainly occurred in early period ${ }^{[30]}$. Therefore, a more multi-dimensional evaluation is of great significance. The functional evaluation methods like QFR may have a promising potential in future evaluation.

This study still has some limitations. First, this is a retrospective single-center observational study with a small sample size, further prospective multicenter cohort studies are needed to verify the findings. Secondly, not all images are suitable for QFR analysis, which may cause the selection bias. Besides, not 
all patients are tested for glycosylated hemoglobin, lack of the glycosylated hemoglobin results makes it hard to assess the long-term level of glucose.

\section{Conclusion}

Compared to patients without T2DM, CAD patients who co-existing T2DM have a worsen QFR value at one-year follow-up and a more obvious decline in QFR within one year. From the perspective of functional evaluation, we corroborate that T2DM can accelerate the functional decline of target coronary vessels after successful PCl.

\section{Abbreviations}

T2DM: Type 2 diabetes mellitus; CAD: Coronary artery disease; PCl: Percutaneous coronary intervention; QFR: Quantitative flow ratio; FFR: Fractional flow reserve; DS: Diameter stenosis; STEMI: ST-segment elevation myocardial infarction; NSTEMI: Non-ST-segment elevation myocardial infarction; TIMI: Thrombolysis in myocardial infarction; LDL-C: Low-density lipoprotein cholesterol; NT-proBNP: N-terminal pro brain natriuretic peptide; CRP: C-reaction protein; MACEs: Major adverse cardiac events; TVR: Target vessel revascularization; LVEF: Left ventricular ejection fraction; BFR: Blood flow resistance; CMD: Coronary microvascular dysfunction; AGE: Advanced glycation end products.

\section{Declarations}

\section{Acknowledgements}

Not applicable.

\section{Authors' contributions}

ZY, QC and JZ contributed equally to this work and share the frst authorship. YL and LiaC contributed equally to this work and share the corresponding authorship. All authors contributed to the study conception and design. Material preparation and data collection were performed by ZY, QC, JZ, LoC, LihC, MY and YY. Data analysis was performed by ZY, QC and JZ. The frst draft of the manuscript was written by ZY, QC and JZ. And all authors commented on previous versions of the manuscript. All authors read and approved the final manuscript.

\section{Funding}

This study was not supported by any funding.

\section{Availability of data and materials}

The datasets generated and/or analysed during the current study are not publicly available due to privacy or ethical restrictions, but are available from the corresponding author on reasonable request. 


\section{Ethics approval and consent to participate}

This study was approved by the Ethics Committee of Union Hospital, Fujian Medical University (No. 2020KY098). Informed consent was waived by the Ethics Committee of Union Hospital, Fujian Medical University, because of the retrospective nature of this study.

\section{Consent for publication}

Not applicable.

\section{Competing interests}

The authors declare that they have no competing interests.

\section{Author details}

${ }^{1}$ Department of Cardiology, Fujian Medical University Union Hospital, No. 29 Xin Quan Road, Fuzhou, Fujian 350001, P.R. China. ${ }^{2}$ Fujian Institute of Coronary Heart Disease, Fuzhou, Fujian 350001, P.R. China. ${ }^{3}$ Fujian Heart Medical Center, Fuzhou, Fujian 350001, P.R. China.

\section{References}

[1] Beckman JA, Creager MA. Vascular Complications of Diabetes. Circ Res. 2016;118(11):1771-85.

[2] Kaur R, Kaur M, Singh J. Endothelial dysfunction and platelet hyperactivity in type 2 diabetes mellitus: molecular insights and therapeutic strategies. Cardiovasc Diabetol. 2018;17(1):121.

[3] Poznyak A, Grechko AV, Poggio P, et al. The Diabetes Mellitus-Atherosclerosis Connection: The Role of Lipid and Glucose Metabolism and Chronic Inflammation. Int J Mol Sci. 2020;21(5):1835.

[4] Cassese S, Byrne RA, Tada T, et al. Incidence and predictors of restenosis after coronary stenting in 10 004 patients with surveillance angiography. Heart. 2014;100(2):153-9.

[5] Kini AS, Kim MC, Moreno PR, et al. Comparison of coronary flow reserve and fractional flow reserve in patients with versus without diabetes mellitus and having elective percutaneous coronary intervention and abciximab therapy (from the PREDICT Trial). Am J Cardiol. 2008;101(6):796-800.

[6] Toth GG, Johnson NP, Jeremias A, et al. Standardization of Fractional Flow Reserve Measurements. J Am Coll Cardiol. 2016;68(7):742-53.

[7] Tonino PA, De Bruyne B, Pijls NH, et al. Fractional flow reserve versus angiography for guiding percutaneous coronary intervention. N Engl J Med. 2009;360(3):213-24.

[8] Cesaro A, Gragnano F, Di Girolamo D, et al. Functional assessment of coronary stenosis: an overview of available techniques. Is quantitative flow ratio a step to the future?. Expert Rev Cardiovasc Ther. 
2018;16(12):951-62.

[9] Tu S, Westra J, Yang J, et al. Diagnostic Accuracy of Fast Computational Approaches to Derive Fractional Flow Reserve From Diagnostic Coronary Angiography: The International Multicenter FAVOR Pilot Study. JACC Cardiovasc Interv. 2016;9(19):2024-35.

[10] Westra J, Andersen BK, Campo G, et al. Diagnostic Performance of In-Procedure Angiography-Derived Quantitative Flow Reserve Compared to Pressure-Derived Fractional Flow Reserve: The FAVOR II EuropeJapan Study. J Am Heart Assoc. 2018;7(14):e009603-.

[11] Xu B, Tu S, Qiao S, et al. Diagnostic Accuracy of Angiography-Based Quantitative Flow Ratio Measurements for Online Assessment of Coronary Stenosis. J Am Coll Cardiol. 2017;70(25):3077-87.

[12] Westra J, Tu S, Winther S, et al. Evaluation of Coronary Artery Stenosis by Quantitative Flow Ratio During Invasive Coronary Angiography: The WIFI II Study (Wire-Free Functional Imaging II). Circ Cardiovasc Imaging. 2018;11(3):e007107.

[13] Spitaleri G, Tebaldi M, Biscaglia S, et al. Quantitative Flow Ratio Identifies Nonculprit Coronary Lesions Requiring Revascularization in Patients With ST-Segment-Elevation Myocardial Infarction and Multivessel Disease. Circ Cardiovasc Interv. 2018;11(2):e006023.

[14] Echavarria-Pinto M, Escaned J, Macías E, et al. Disturbed coronary hemodynamics in vessels with intermediate stenoses evaluated with fractional flow reserve: a combined analysis of epicardial and microcirculatory involvement in ischemic heart disease. Circulation. 2013;128(24):2557-66.

[15] Camici PG, Crea F. Coronary microvascular dysfunction. N Engl J Med. 2007;356(8):830-40.

[16] Beckman JA, Creager MA, Libby P. Diabetes and atherosclerosis: epidemiology, pathophysiology, and management. Jama. 2002;287(19):2570-81.

[17] Reith S, Battermann S, Hellmich M, et al. Impact of type 2 diabetes mellitus and glucose control on fractional flow reserve measurements in intermediate grade coronary lesions. Clin Res Cardiol. 2014;103(3):191-201.

[18] Gargiulo G, Stabile E, Ferrone M, et al. Diabetes does not impact the diagnostic performance of contrast-based fractional flow reserve: insights from the CONTRAST study. Cardiovasc Diabetol. 2017;16(1):7.

[19] Smit JM, El Mahdiui M, van Rosendael AR, et al. Comparison of Diagnostic Performance of Quantitative Flow Ratio in Patients With Versus Without Diabetes Mellitus. Am J Cardiol. 2019;123(10):1722-8.

[20] Lee JM, Choi KH, Koo BK, et al. Comparison of Major Adverse Cardiac Events Between Instantaneous Wave-Free Ratio and Fractional Flow Reserve-Guided Strategy in Patients With or Without Type 2 
Diabetes: A Secondary Analysis of a Randomized Clinical Trial. JAMA Cardiol. 2019;4(9):857-64.

[21] Hu X, Zhang J, Lee JM, et al. Prognostic impact of diabetes mellitus and index of microcirculatory resistance in patients undergoing fractional flow reserve-guided revascularization. Int $\mathrm{J}$ Cardiol. 2020;307:171-5.

[22] Haas AV, McDonnell ME. Pathogenesis of Cardiovascular Disease in Diabetes. Endocrinol Metab Clin North Am. 2018;47(1):51-63.

[23] Schmidt AM. 22016 ATVB Plenary Lecture: Receptor for Advanced Glycation Endproducts and Implications for the Pathogenesis and Treatment of Cardiometabolic Disorders: Spotlight on the Macrophage. Arterioscler Thromb Vasc Biol. 2017;37(4):613-21.

[24] Corpus RA, George PB, House JA, et al. Optimal glycemic control is associated with a lower rate of target vessel revascularization in treated type II diabetic patients undergoing elective percutaneous coronary intervention. J Am Coll Cardiol. 2004;43(1):8-14.

[25] lakovou I, Schmidt T, Bonizzoni E, et al. Incidence, predictors, and outcome of thrombosis after successful implantation of drug-eluting stents. Jama. 2005;293(17):2126-30.

[26] Madonna R, Balistreri CR, Geng YJ, et al. Diabetic microangiopathy: Pathogenetic insights and novel therapeutic approaches. Vascul Pharmacol. 2017;90:1-7.

[27] Di Carli MF, Janisse J, Grunberger G, et al. Role of chronic hyperglycemia in the pathogenesis of coronary microvascular dysfunction in diabetes. J Am Coll Cardiol. 2003;41(8):1387-93.

[28] Kassaian SE, Goodarzynejad H, Boroumand MA, et al. Glycosylated hemoglobin (HbA1c) levels and clinical outcomes in diabetic patients following coronary artery stenting. Cardiovasc Diabetol. 2012;11:82.

[29] Nozue T, Yamamoto S, Tohyama S, et al. Statin treatment for coronary artery plaque composition based on intravascular ultrasound radiofrequency data analysis. Am Heart J. 2012;163(2):191-9.e191.

[30] Lee CH, Choi SW, Jun SW, et al. Clinical impact of diabetes mellitus on 2-year clinical outcomes following PCl with second-generation drug-eluting stents; Landmark analysis findings from patient registry: Pooled analysis of the Korean multicenter drug-eluting stent registry. PLoS One. 2020;15(6):e0234362.

\section{Figures}




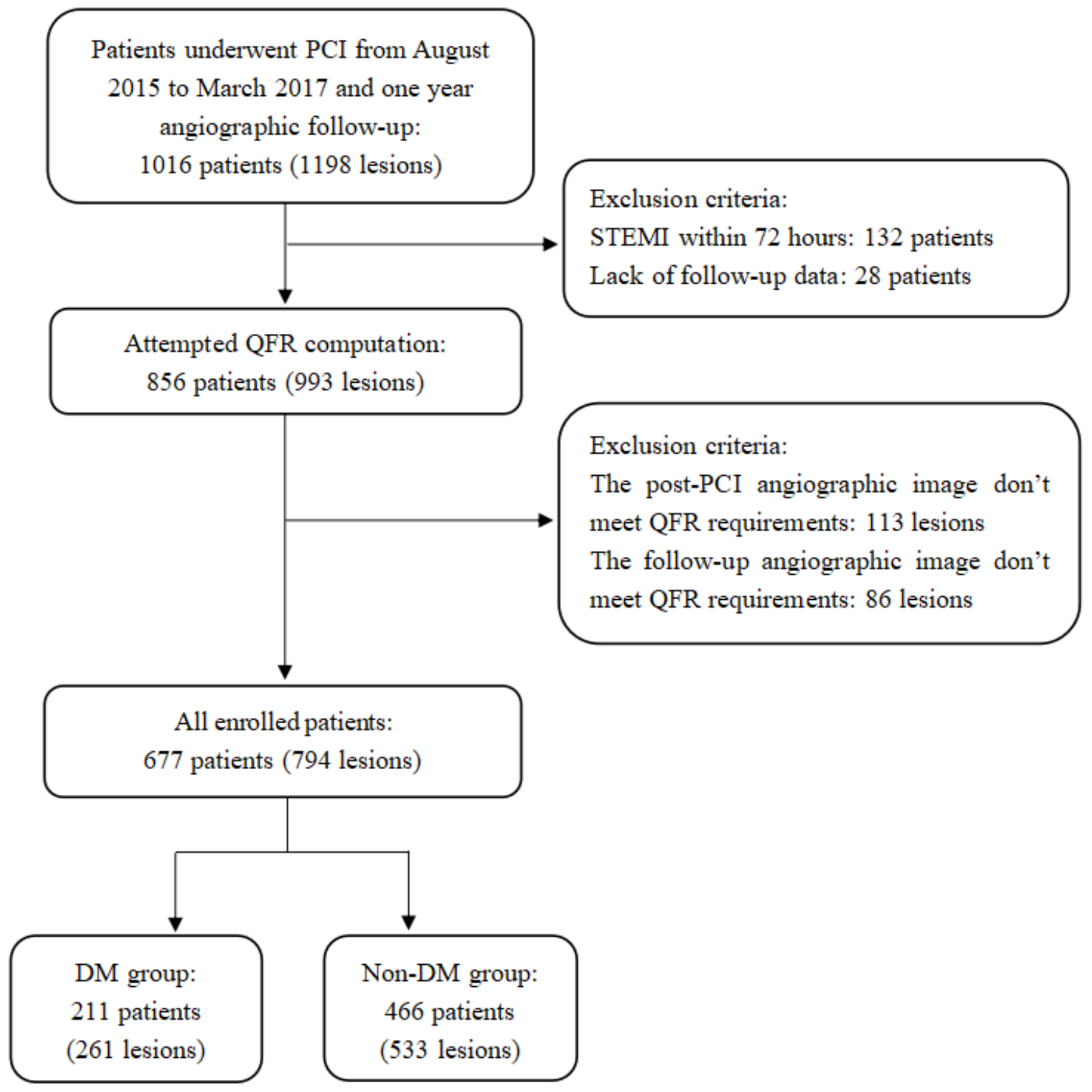

Figure 1

Flow chart. PCl, percutaneous coronary intervention; STEMI, ST-segment elevation myocardial infarction; QFR, quantitative flow ratio; DM, diabetes mellitus 
A

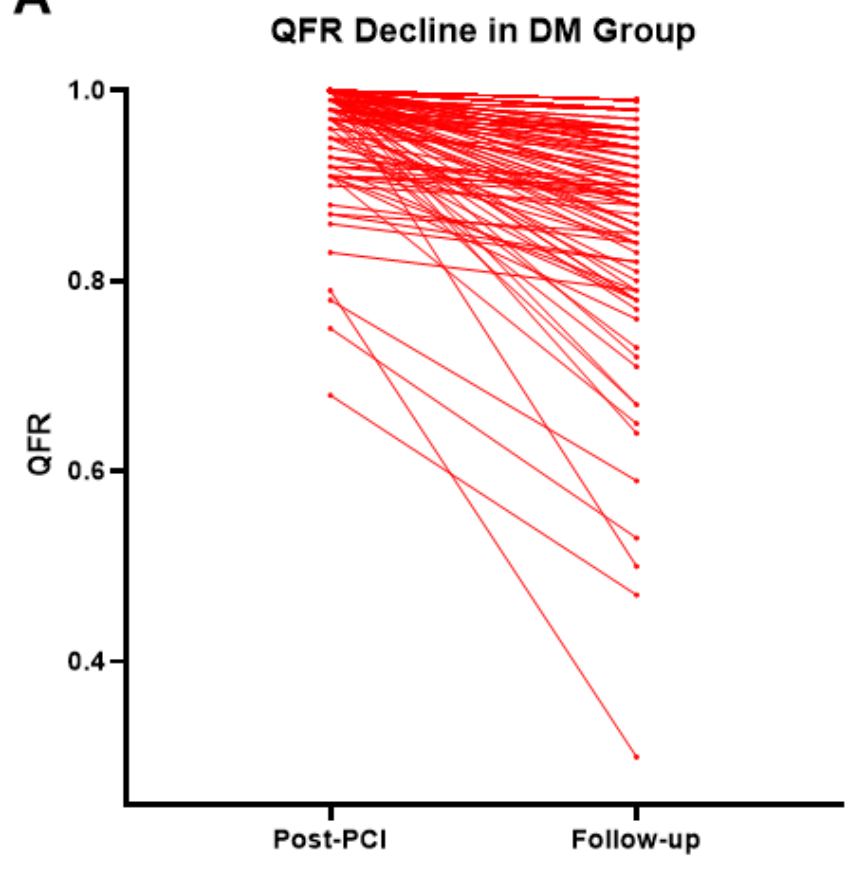

B

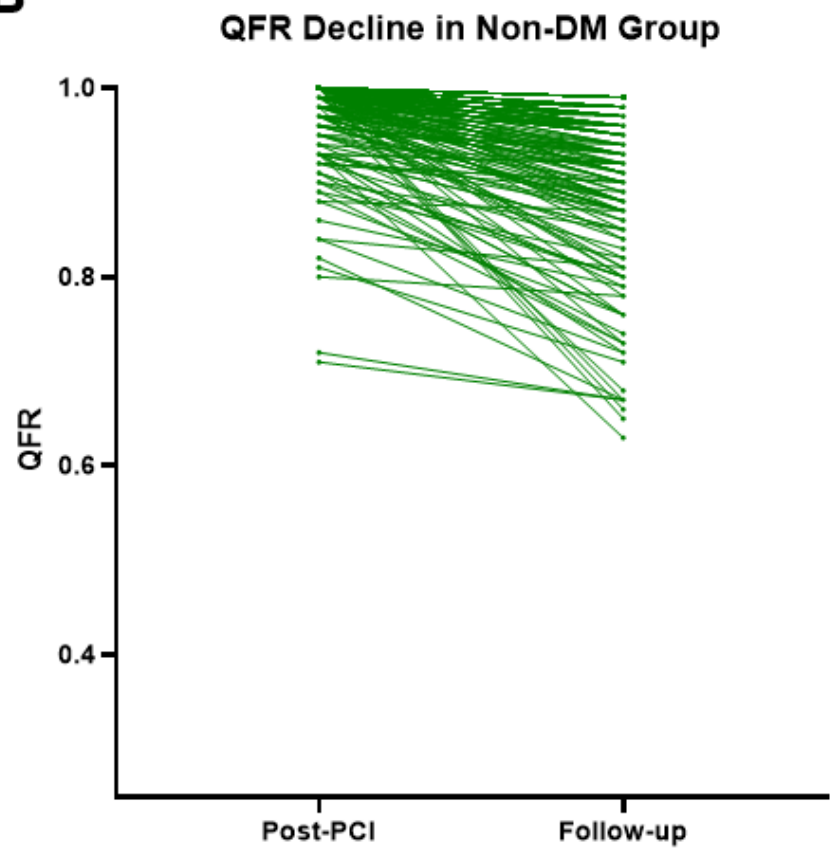

Figure 2

The situation of QFR decline in the DM group (A) and Non-DM group (B). QFR, quantitative flow ratio; DM, diabetes mellitus; $\mathrm{PCl}$, percutaneous coronary intervention 